\title{
Virtual Teaching And Learning Challenges- An online learning experience with UAE students
}

\author{
Mawdudur Rahman \\ Professor of Accounting, Suffolk University,USA
}

Learning and Teaching in Higher Education: Gulf Perspectives

Vol 3 No 1, January 2006

\begin{abstract}
In this paper we provide evidence of successful online learning experience with UAE students at Zayed University in the UAE. First we describe the processes and technologies used in delivering the online course "Virtual Business Challenges". Based on survey results we discuss students' evaluations and experience and the technologies used. Overall, students had a positive learning experience in the course.
\end{abstract}

\section{Background}

In the debate about the effectiveness of online education, scholars have shown that online education can provide a quality learning experience (Russell, 1999; Evans and Haase, 2001; Schoech, 2000; Conrad, 2002; Fitzpatrick, 2002). In this paper we provide further evidence of successful online learning which transcends cultural boundaries and presents an example of an effective e-learning experience with students in the United Arab Emirates (UAE).

Teaching an online course is a challenge. Online learning and teaching have placed new demands on students and teachers, and Schrum and Hong (2002) provide a list of dimensions for online learners and educators that underlie positive learning experiences. The seven dimensions they identified and confirmed through their research are: 1) access to tools, 2) technology experience, 3) learning preference, 4) study habits and skills, 5) goals and purposes, 6) life style factors and 7) personal traits and characteristics. Among the strategies Schrum and Hong recommend are: students' posting biographies; frequent interactions; collaboration; participation; question asking forums; topical flexibility, and minimizing technology requirements.

In the online mode the students are not in a classroom and the teacher is not managing the class face-to-face (f2f); thus opportunities to create a captive class environment through charisma and threat are virtually absent. The expression of personal emotions is reduced to a few symbols and notations for the online classrooms, audio-visual live interactions and feedback typically do not exist, and verbal and non-verbal communication is severely limited by the capability of the technology. McDonald (2002) argues that while these are limiting factors for some participants, many studies have found positive outcomes in online interactions: "more students respond, responses are longer and more complex, and interactions are increased in online education when compared to face to face."

Factors contributing to students' participation in synchronous online chat discussion sessions include the following:

1. Students find the online chat environment non-threatening because there is no physical presence involved. Also the student is usually participating from his/her own 'home environment'

2. One or two students do not dominate the chat discussion. (A well structured chat is guided by a chat protocol (see Appendix -3 ) to ensure fair chances for everyone in the class.

3. Good chat protocol does not allow lengthy contributions by one single person at one time. Students can write one to two short sentences to add their comments or to ask questions.

4. New students quickly learn the appropriate chat culture from a qualified and trained faculty member,

5. Involved chat participation needs faculty support and preparation, such as the posting of chat topics and subtopics before the chat session so that students come prepared to participate.

6. Chat must be graded as class participation. It should count for at least $20 \%$ of the final grade.

To provide successful online education it is essential to understand how the limiting and the supporting factors affect the learners, educators, and the programs. There is a continuing debate about the effectiveness of online teaching and learning (Fox, 1998; Sonner, 1999; Dutton et al., 1999). Much to the credit of the online educators and researchers, a substantial 
amount of empirical research evidence is now available to show that effective education is possible through online media. Russell (1999) reviewed 355 research reports, summaries and papers and found that online and face-to-face learning can provide equally effective education. Well known colleges and universities around the globe are offering courses in social sciences, business subjects, engineering, and basic sciences which do not require hands-on laboratory experiences.

It is now well accepted that online learning is a credible paradigm (Eastman and Swift, 2001). Online education is learner driven and not faculty driven (Rahman, 2001). Online students learn differently (Dutton, 2002), and successful distance learners exhibit higher motivation and self-discipline in achieving their learning goals (Cookson, 1989). Online education provides an alternative way of teaching and learning which works for a particular group of educators and learners. Effective learning is possible through online media with the right mix of educators, learners, and tools.

In line with the above discussions, the rest of this paper provides empirical evidence from an online course offered in the UAE, which demonstrates how an effective online course can be designed and delivered. It is shown how the students and the instructor interacted in synchronous and asynchronous modes to support effective learning.

\section{Background}

In 2004-5 a course entitled Virtual Management Challenges (VMC) was offered as an online course to third and fourth year undergraduate business students at Zayed University, UAE. Zayed University (ZU) is an urban university with two campuses, located in Dubai and Abu Dhabi, and was established in 1998 to educate UAE national women. Currently there are 3000 students in five colleges and a number of Outreach Centers at ZU. It is a bilingual University, with an extensive English Language Program. All students, faculty, and administration are provided with laptops by the university, and internet connections are available all over the campus at each classroom seat. Students are proficient in using Blackboard, which is used to some extent in most courses at the university.

The VMC course was offered online because the college needed a two credit course for some of its graduating students and the author suggested to offer the course online. Students gave the following reason for taking the online VMC course: "It is a new learning experience; I heard many advantages of online learning; it is more interesting; and to have a new experience." The objectives of the course are to make students aware of the challenges faced by today's organizations because of changes in technology; to develop leadership skills in virtual teams; and to recognize the characteristics of "built-to-last" companies.I had offered this course previously at Suffolk University, Boston, where it was well received by the students. For Suffolk students the course was on the iDLSystems platform, and covered three specific areas; 1) virtual teams, 2) trends in information technology and 3) management challenges for tomorrow's organizations. In both iterations these areas were organized as three units, and course assignments included two case studies (Sub-Arctic Survival case and CISCO case), three discussion topics(see appendix 1) and three live chat sessions (see appendix 2) to complete over a six week period.

\section{The process}

The course was delivered at ZU using two learning management systems (LMS platforms). We used Blackboard for communication, discussion, and feedback and iDLSystems for content delivery and tests. iDLSystems is a hosted platform and Blackboard is an in-house server managed system. The original course at Suffolk University was on iDLSystems where it was offered to test the effectiveness of learning style-oriented LMS in online education. In the $\mathrm{ZU}$ course we did not want to transfer materials from iDLSystems to Blackboard for several reasons, including web standards incompatibility, ZU student familiarity with Backboard, efficiency of chat functions in Blackboard vs. iDLSystems, and more effective content presentation in iDLSystems. Consequently students participated in the discussion board and synchronous chat sessions using Blackboard, while the reading materials and tests were included in iDLSystems, whereby students were given a choice of using learning materials presentations based on their learning styles. The learning materials were presented in three styles, 1) apprenticeship, 2) discovery and 3) case method.

- Apprenticeship:

Students learn step-by-step, following the lead of the instructor

- Discovery:

Student learns by conducting experiments, then analyzing the results

- Case method

Student learns through case studies or storytelling

The content was developed by the course instructor and the organization and presentation were prepared by experts from iDLSystems. Students were allowed to repeat the test on any 
unit only three times to improve their scores. At the end of each test an analysis of students' scores was made by the system, and the system would not open the next unit to the student until the student achieved a passing score in the current unit.

There were ten students on the course, divided into two teams. They chose the team names and the team leaders: one team called themselves Burjuman and the other Wafi (names of famous local shopping malls) The teams met in chat rooms specified for their case discussions. The students were familiar with Blackboard navigational tools, and the university's IT Helpdesk answers all technical questions from the students. iDLSystems, on the other hand, was a new platform for the students, but they did not face any major difficulty in using it and were able to meet all the course requirements without major difficulty. The providers of iDLSystems maintain their own online helpdesk.

\section{Course Organization}

As indicated above, the course used two LMSs: iDLSystems and Blackboard. The course web pages on both the systems provide side panels with buttons to organize different course elements. To view the course web page on iDLsystems see appendix 6.

\section{Syllabus:}

A fairly detailed syllabus was used with clearly stated expectations, grading policy, relevant dates, chat time, and assignments, and was available to students online before the start of the course. The syllabus includes the subjects which are covered in each unit, with dates and the week of the unit. It also included the instructor's bio, with links to some of the instructor's online articles. The syllabus was posted on both Blackboard and iDLsystems websites.

\section{Student introductions:}

Students submit their introduction in this section of Blackboard before the start of the course. Students need to know each other at the start of the course so that they know the class mix and each other's background. This helps the instructor to understand the comments and questions raised by a student, and also helps students while working in a team.

\section{Course content:}

The main course content is divided into three units in IDLsystems, which are delivered over six weeks. The course content was presented in iDLSystems.

Unit contents and the learning process:

The course includes one introductory unit and the three main content units. Each unit is scheduled for one week and includes lecture notes, power point slides, and audio to describe the contents. Students can read the lecture notes, hear the audio with the PowerPoint slides, and take the quiz, all of which are posted on the iDLSystems. Outlines of contents included in each unit are listed below:

Unit 1 is the introduction to the online platforms and how to navigate through different menu items.

Unit 2 (Team Building) describes the concept of a team, team development, and leadership in virtual teams

Unit 3 (Information Technology) describes changes and challenges in IT, the impact of IT on organizations and IT and corporate strategy

Unit 4 (Continuity and Change) discusses visionary companies, paradoxes of our times, characteristics of "built-to-last" companies, needs to change and continue, future organizations, and how to prepare for a career in a future company.

The course also included asynchronous threaded discussions and synchronous text chat discussions in Blackboard.

\section{Interactions}

Opportunities for interactions are essential components for online learning both in synchronous and asynchronous modes, as for distance learning more generally (Daniel and Marquis, 1979; Picciano, 2002). Dean (1994) considers interactivity as a critical characteristic of online education; interactions provide students and the instructor with opportunities to share their knowledge, learn from each other, and test ideas and theories in a group setting. The instructor learns about students' progress on a continuous basis and areas of strength and weakness of the students, thus contributing to the overall effectiveness of the online learning experience. Interaction opportunities in the course have been provided through the use of synchronous chats, threaded discussions, and emails. We later found students also used mobile telephony and SMS for interactions between them.

a) Emails:

The class and the instructor communicate with anyone in the class using the email facility within Blackboard. The turn around time from the instructor is 24 hours.

b) Text Chat 
Chat is a synchronous interactive tool. The class meets online for an hour and a half at a scheduled chat time like a regular class meeting, but the location is up to the individual: one student had a young baby, and the online format enabled her to participate where she could not have otherwise. Chat is implemented though Blackboard, and attendance in the chat is compulsory. The instructor circulates chat topics a few days before the session and students prepare for discussions using materials from iDLSystems. The chat protocol (Appendix 3 ) sets ground rules for the chat process. Chat discussions are graded and archived for future viewing.

c) Discussion board

A discussion board is an asynchronous interactive tool which students use in Blackboard environment. A discussion board topic remains open for a week and all students are required to participate. Discussion board participation is governed by a protocol (Appendix 4) and is a gradable item

\section{d) Course Documents}

As previously described, course materials uploaded in iDLSystems include assignments, tests, reading, and PowerPoint slides. Additional documents are uploaded to Blackboard by the instructor. Students were also allowed to upload documents to Blackboard.

e) Tests and Quizzes

Tests and quizzes are set online in iDLsystems for automated grading, comments, and corrected answers. There are time limits and password controls; students can take a test twice only. The tests are in multiple-choice format and are remedial in nature. The systems provided instant feedback to the students on their learning level and then guided them to remedial activities with suggested readings for their wrong answers so that they can focus on their weak areas.

f) Announcements ( Message board)

The announcement window in Blackboard is used to communicate important latest information to the class.

\section{Data Collection}

Several data sources were used to prepare this paper. Data were collected through self reported attitudinal surveys. At the beginning of the course students completed a questionnaire on their background and reasons for taking the course, and made comments on their attitudes to online learning; at the end of the course students also completed a 26 item standardized course evaluation instrument (CEI - see appendix 5).

A segment of data was collected from the course process as the course was going on. For example, all chat and discussion board records were archived and analyzed qualitatively and quantitatively. Screenshots are shown in the appendices to demonstrate the nature of the data, which are available from chat and discussion board archives.

\section{Background Data}

Of the 10 students in the class, this was the first online course for nine. Six of the students are in their $3 r d$ year and four are in their 4 th year of a 5 year college program. The GPA of the students ranges from 2 to 4 and above, as reported below:

\begin{tabular}{|l|l|l|}
\hline GPA & Percentage & Response \\
\hline 2 and above & 33.3 & 3 \\
\hline 3 and above & 44.4 & 2 \\
\hline 4 and above & 22.2 & 2 \\
\hline Total & & 9 \\
\hline
\end{tabular}

1. Discussion board evaluation (asynchronous discussion)

The discussion board is an asynchronous discussion and provides evidence of students' participation. Some students participate very vigorously while others do not see value in discussion board participation. Students participate in a discussion throughout the week. Students' evaluation of thread discussions for the course is 4.2 on a 5 -point scale as in the table below.

\begin{tabular}{|l|l|}
\hline Course & Ave. score (5-point scale) $n=10$ \\
\hline ZU course & 4.2 \\
\hline
\end{tabular}

\section{Chat discussions evaluation}

As said before, chat is a synchronous interaction class where students meet every week for one hour and a half. 
Below are summary statistics from the course chat logs.

\begin{tabular}{|c|c|c|}
\hline Number of students & 10 & \\
\hline Number of chat sessions & 3 & \\
\hline $\begin{array}{l}\text { Total number of meaningful responses for the three } \\
\text { sessions ( session 1:182 + session } 2: 437+\text { session } 3: 646 \text { ) } \\
=\end{array}$ & 1262 & \\
\hline Average number of responses per student in all chats & 162 & $\begin{array}{l}50.4 \% \text { of total } \\
\text { professor's } \\
\text { entries }\end{array}$ \\
\hline \multicolumn{3}{|l|}{ Average number of responses per chat session: } \\
\hline Chat session 1 & 26 & \\
\hline Chat session 2 & 54 & \\
\hline Chat session 3 & 81 & \\
\hline Total number entries by the professor in the three session & 321 & \\
\hline Average number of entries by the professor & 107 & \\
\hline Highest number of responses for a chat session & 171 & \\
\hline Lowest number of responses for a chat session & 3 & \\
\hline
\end{tabular}

The table above reveals that at the first chat session students did not get much into it but as they got accustomed to it and understood the process and the value of participation they got more involved. As we see from the above table, on average each student's participation is $50.4 \%$ of the average faculty participation in the chats. The data suggest that the professor has to remain active to keep students active in online chats.

\section{Students' course evaluation and feedback}

A 26 item survey questionnaire (available on request from the author) was administered at the end of the semester to get feedback from the students. The average scores of the survey responses by the students are included in appendix 5 . Students scored each question from one to five on a Likert scale. When the 26 questions are ranked as to the scores assigned we see interesting relationships. Three clusters emerge as the students' perception of the importance and usefulness of the elements in the process of online learning (see below).

One must be careful not to generalize across cultures from these tendencies for two reasons; 1) cultural differences and 2) the small size of the sample. We did not measure any cultural difference factors; however I would say in comparison with the Suffolk group that UAE students (a) are more dependent on the professor, so need more opportunity to interact, (b) are more homogenous as to technology experience, so we spent less time on technology glitches, (c) are more focused because they invest more time in their courses, (d) often suffer from "group-think" syndrome (i.e. due to less divergence among team members they fail to come up with alternative solutions to choose from: everyone accepts what one proposes), (e) try hard (and sometimes unsuccessfully) to overcome language deficiencies, ( $f$ ) spend time on personal greetings as an important part of the discussions in chats and threads, and $(\mathrm{g})$ use local terms in Arabic to comment to each other in the chat sessions, reducing their practice of English.

The three clusters referred to above are: people related variables (about the student and the instructor), process related variables, and technology related variables; these could be characterized as 1) I am feeing good, 2) I am positive about the process, and 3) I am technology transparent. The clusters are formed heuristically, based on ranked scores of the questionnaire items: no statistical tools were used to create the clusters because of the small sample size. Below is a discussion of the three clusters.

\section{I am feeling good}

This is the highest ranked cluster. The average score is 4.67 out possible 5 . Student must feel good about what is going on in the online course and with the instructor., which is a big responsibility. Online education is not an impersonal and technology dominant delivery. When successful, it is a result of the blending of the students, instructor and the instructional materials interface that achieve success. 
I am learning how to think clearly about the course 4.6

subject matter

The class is well organized

The instructors are easy to contact

4.6

The level of detail provided is about right

Overall, the instructors are doing an excellent 4.7 teaching job

\section{I am positive about the process}

The students' evaluation of the technology and the process elements needs to be positive. In this case the students are already very familiar with Blackboard and learnt how to use iDLSystems. They think technology, process, and the course loads are important but not as high as their overall feeling about the instructor and their self engagement. The overall average score is 4.1 , which is noticeably different from "I am feeling good".

Case write-ups are valuable

Student's value each other's opinion

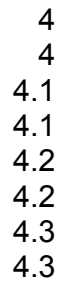

Online chat sessions are valuable

With Blackboard I learnt materials easily

The course workload is about right

The threaded discussions are useful

Email is a valuable course tool

With iDL systems I learnt materials easily

\section{I am technology transparent}

The third cluster is about technology. The students showed somewhat neutral (indifferent) attitude towards technology. The technology is not a perceived obstacle for these students, which may not be true in other cases. These students are already very conversant with Blackboard and had previously very good IT training. Therefore, technology is not at all a burden on their eLearning. Technology has been transparent in the process. Students assigned a low score for the suggestion to use only one of the two platforms; what this means is that they found the use of both platforms value-added in the process. Probably, one reason was that we combined the best of both systems (flexible content delivery through IDLsystems and interaction through Blackboard). The other side of the coin is that the use of technology is not enough to deliver a successful online course, at least in this culture, where human interactions are valued highly. The average score for the cluster is 3.55 , which is the lowest of the three clusters.

I recommend the use of iDL systems only

I recommend the use of Blackboard only

2.9

iDlystems is user friendly

3.8

Blackboard is user-friendly

\section{Summary and Conclusion}

Online education can provide a quality learning experience. In this paper we provide further evidence of successful online learning which transcends cultural boundaries, and present an example of effective learning experiences with UAE students. Many studies have been done in Western cultures to show that effective learning is possible via online media. The present study showed that the same conclusion is valid in Arab cultures if one is careful in designing the course by keeping in mind language constraints and social values.

The paper briefly discussed, along with relevant literature, that the online learning process is different from face-to-face learning, and effective learning is possible through online media. The author observes that the online learning experience depends on the learners' motivation, technologies used, processes applied, and institutional capacity to provide adequate faculty and other resources. Next, we provided information on the processes used for the delivery and organization of online courses. An overview of the technologies used and the interaction processes followed. In the third section we discussed the evaluation results of the tools used and the end of the semester course evaluations.

Within a short span of time online education has come a long way. UAE universities are making significant efforts to expand online teaching at different levels. Our experiment in this course showed that one has to be careful before accepting online course models and technologies and should be prepared to make variations depending on the students' needs and cultural constraints UAE students and perhaps also other Arab students need different types of course design, delivery, and interactions than students in other countries, such as North America. In this course at least, students are more concerned with their personal satisfactions with the process and the instructor than with the technology. 
Two final conclusions we want to draw from this study are that technology cannot replace the need for an instructor who is sensitive to students' needs, and who can blend the technology applications, interactions, and his/her knowledge of the subject matter effectively. Secondly, blending of different elements in the process into a smooth seamless whole which matches students' cultural expectations is necessary for effective online teaching and learning.

AUTHOR'S NOTE: The author gratefully acknowledges the contributions made by the editor and the anonymous reviewer through their comments and editorial suggestions.

\section{Bibliography}

Conrad, D.L. (2002). Engagement, Excitement, Anxiety, and Fear: Learners' Experience of Starting an Online Course. The American Journal of Distance Education, 16(4), 205-266.

Cookson, P.S., (1989). Research on learners and learning in distance education: A review. The American Journal of Distance Education, 38:151-161.

Daniel, J. and Marquis, C. (1979). Interaction and independence: getting the mixture right. Teaching at a Distance: 15, 25-

44.

Dean, L. (1994). Telecomputer communication: The model for effective distance learning. ED Journal, 8(12) J-1-J-9.

Dutton, J., (2002). How Do Online Students Differ From Lecture Students? Journal of Asynchronous Learning Networks, 6(1), 1-20.

Dutton, J., M. Dutton and J. Perry. 1999. Do Online Students Perform As Well As Traditional Students?, in The No Significant Difference Phenomenon ed. T. L: Russell. IDECC, Montgomery.

Eastman, J.K., and Swift, C.W. (2001). New horizons in distance education, Journal of Marketing Education, 23(1): 25-35.

Evans, J.R., and Haase, I. M., (2001). Online Business Education in the twenty-first century: an analysis of potential target markets. Internet Research: Electronic Networking Applications and Policy, 11 (3) :246-260

Fitzpatrick, R., (2002). Is distance education better than the traditional classroom? Accelepoint www.clearpoint.com/accelepoint/articles/r fitzpatrick 060101.shtml

Fox, J., (1998). Distance education: Is good enough? The University Concourse, (3):3-5.

McDonald, J., (2002). Is "as good as face-to-face" as good as it gets? Journal of Asynchronous Learning Networks, 6(2), 1023.

Picciano, A.G., (2002). Beyond Student Perceptions: Issues of interaction, presence, and performance in an online course,

$$
\text { Journal of Asynchronous Learning Networks, 6(1). }
$$
http://www.aln.org/publications/jaln/v6n1/v6n1_picciano.asp

Rahman, M., (2001). Faculty recruitment strategies for online programs. Online Journal of Distance Education, Winter, 4(4) 2001. http://www.westga.edu/ distance/ojdla/winter44/rahman44.htm

Russell, T.L. (1999). The No Significance Phenomenon: A Comparative Research Annotated Bibliography on Technology for Distance Education. (Office of Instructional Telecommunications, NCSU Box 4701, Raleigh, NC 27695-7401).

Schoech, D., (2000). Teaching over the internet: Results of one doctoral course. Research on Social Work Practice 10(4):467-486

Schrum, L. and Hong, S., (2002). Dimension and Strategies for Online Success: Voices from experienced educators. Journal of Asynchronous Learning Network, 6(1).

Sonner, B., (1999). Success in the capstone business course-assessing the effectiveness of distance learning. Journal of Education for Business, 74(4):243-248.

Wan, G. (2001) The Learning Experience of Chinese Students in American Universities: A cross-cultural perspective. 
Appendix -1

Discussion Topics on the BlackBoard

COURSES $>$ BUS-395-502-SPRI-200422-3--INDEPENDENT STUDY... $>$ DISCUSSION BOARD

Discussion Board

\section{Add Forum}

\section{$1 \quad$ Built to Last}

Was Shaikh Zayed (Yarhamu Allah) a clock builder or a time teller? Support your positon by examples and arguments. Are there any companies in UAE which will qaulify to become built-to-last companies. This thread will close on April 23rd.

\section{$2 \quad$ IT revolution and the spinning wheel of paradox}

Tech-revolution have changed our life styles, our thinking, behavior, actions, cultures, education, politics, and social life. The IT has offered us the choices to be simple and complicated, broader and narrower, global and local, insular and open-minded- we are living in an age of paradox (words/phrases meaning two opposite senses - like one can be simple and also complicated, better quality but lower costs etc.). Our boundary is continuously spanning (expanding) in a spinning (going around) wheel of paradox. In fact, we are living in a future knowledge dependent ( more information needed to succeed) society.

\section{Cutural Dimension- doing business in Dubai}

Cultural dimensions of team building

Are there cultural implications to team building? Explain how the concept of teams differs in a country about which you have some

\section{Appendix -2}

\section{An excerpt from chat session -3}

It high lights a) cultural context b) some students join late c) connectivity remains a problem

Fatima Fatima Ahmed Mohamed Ahmed: AND you have to come up with a decsion that gather the best of all choices Apr 23, 2005 7:26:04 PM GST

Hessa Hessa Abdulla Miran Mohammad: Salamz .. Apr 23, 2005 7:26:09 PM GST Mawdudur Rahman: SO DO NOT THINK COST OR QUALITY- THINK ABOUT HI QUALITY WITH LO COST Apr 23, 2005 7:26:09 PM GST

Lamya Lamya Ebrahim Abdulrahman Abdulkari: it says that you can change OR be stable Apr 23, 2005 7:26:11 PM GST

Khuloud Khuloud Ahmed Taimour Mirza Ali: wa3lekom Apr 23, 2005 7:26:17 PM GST

Mawdudur Rahman: HHA- OALAIKUM ASSALAM Apr 23, 2005 7:26:23 PM GST

Suad Suad Abdulla Hassan Jaffar Al Saffa: w3aleeeekom el salaaaam Apr 23, 2005 7:26:39

PM GST

Hessa Hessa Abdulla Miran Mohammad: so wats up ?? Apr 23, 2005 7:26:40 PM GST

Lamya Lamya Ebrahim Abdulrahman Abdulkari: w3leekom esalam :) Apr 23, 2005 7:26:47 PM GST

Salha Salha Rashid Mohamed Hassan Buloush: what about high quality with low cost ? Apr 23, 2005 7:26:47 PM GST

Khuloud Khuloud Ahmed Taimour Mirza Ali: yes ??? Apr 23, 2005 7:26:57 PM GST

Hessa Hessa Abdulla Miran Mohammad: erbeeeeeeee3ti :) Apr 23, 2005 7:26:58 PM GST

Mawdudur Rahman: YOU SHOULD WORK TO GET HI QULIY AND NOT INCREASE COST Apr 23, 2005 7:27:11 PM GST

Lamya Lamya Ebrahim Abdulrahman Abdulkari: Iol halla Apr 23, 2005 7:27:21 PM GST 
Fatima Fatima Ahmed Mohamed Ahmed: fatima atiq is having problems getting to the chat, she is trying hard Apr 23, 2005 7:27:22 PM GST

Khuloud Khuloud Ahmed Taimour Mirza Ali: ???????????????? Apr 23, 2005 7:28:01 PM GST

Mawdudur Rahman: WE WANT BOTH LO COST AND HI QUALITY AND NOT THE OTHER WAY Apr 23, 2005 7:28:03 PM GST

Asma Asma Rashid Dafoos Rashid Almehairi: the same problem i had before Apr 23, 2005 7:28:06 PM GST

Hessa Hessa Abdulla Miran Mohammad: i dont think its easy to get a product or service with a high quality and low cost .. you can find a one with high quality and good price not low Apr 23, 2005 7:28:07 PM GST

Salha Salha Rashid Mohamed Hassan Buloush: $\mathrm{i}$ think always quality is related to hi cost Apr 23, 2005 7:28:27 PM GST

Mawdudur Rahman: HHA- WE MEAN GOOD PRICE Apr 23, 2005 7:28:34 PM GST

Hessa Hessa Abdulla Miran Mohammad: 3n sho yermes ?? Apr 23, 2005 7:28:34 PM GST

Khuloud Khuloud Ahmed Taimour Mirza Ali: yes u r right HHas Apr 23, 2005 7:28:40 PM GST

Lamya Lamya Ebrahim Abdulrahman Abdulkari: its not always SSR Apr 23, 2005 7:28:40 PM GST

Suad Suad Abdulla Hassan Jaffar AI Saffa: i agree HHA Apr 23, 2005 7:28:41 PM GST

Mawdudur Rahman: PICK ANOTHER EXAMPLE WITH OR AND AND Apr 23, 2005 7:28:49 PM GST

Hessa Hessa Abdulla Miran Mohammad: i meant thats not high and not low .. that most people can afford Apr 23, 2005 7:29:01 PM GST

Khuloud Khuloud Ahmed Taimour Mirza Ali: yes sir sometimes it's difficult to have haigh $Q$ and low cost Apr 23, 2005 7:29:11 PM GST

Asma Asma Rashid Dafoos Rashid Almehairi: if the quilty is good the price will be high alwayes Apr 23, 2005 7:29:12 PM GST

Hessa Hessa Abdulla Miran Mohammad: thanx saud :) Apr 23, 2005 7:29:12 PM GST

Suad Suad Abdulla Hassan Jaffar Al Saffa: because the hi quality cost much thats why the price is hi not low otherwise they will lose Apr 23, 2005 7:29

\section{Appendix -3}

\section{Online Chat Protocol}

We want to insure that your online experience is as interactive and beneficial as possible, so please take a moment to review the following outline for chat room participation, or chat protocol.

Please make every effort to attend the chat rooms; if unavailable make sure to inquire with your professor directly as to how to make up for the missed chat session. Chatroom attendance is mandatory. In a special situation the faculty member can make an exception.

Several elements of chat protocol are important:

- Session time:

Please enter the chat room session on time.

- Multiple, simultaneous topics:

Try to avoid multiple, simultaneous topics - since many people may respond at the same time, it is confusing to have several different discussions ongoing.

- Personal discussions:

If something is important to say to someone, use the private message facility, not the main chat facility.

- Avoid off topic asides:

Something triggered by the chat discussion, but which has nothing to do with the issue under discussion.

- Flames:

Treat all class members with respect, these are working sessions, stay focused on the subject matter.

- Sentences/Comments:

To help ensure efficient posting of your comments, use short sentences. If you are addressing a specific individual, begin your comment with that person's initials.

- Typos:

Because of the spontaneous nature of chat sessions, typographical, spelling, and grammatical errors will be condoned. The ability to respond quickly is very important to a lively discussion.

All classroom codes of conduct prescribed by Zayed University will apply in the case of dysfunctional behavior in the chatrooms. Any disruptive activity will result in serious 
disciplinary action; this may amount to suspension, an ' $F$ ' grade, or dismissal from the program.

Different faculty may approach chat sessions in different formats. Do not expect homogeneity in the manner in which faculty use chat sessions.

\section{Appendix -4}

\section{Discussion Board Protocol}

Any one message must not exceed 120 words or ten lines. A second message by the same person will not exceed 60 words. A person should not submit more than three messages in one topic. Participation is graded based on the quality of discussions, the relevance to the topic, new ideas and examples, and references to current topics or one's own experience. Other important considerations include good communication style as adhered to in business writing, and conciseness.

Topics must be completed within the week and late messages are not graded.

\section{Appendix -5}

The comparative results of the 26 item end of the term evaluation

\begin{tabular}{|c|l|l|}
\hline & \multicolumn{1}{|c|}{ Items } & \\
\hline $\mathbf{1}$ & The class is well organized & 4.6 \\
\hline $\mathbf{2}$ & $\begin{array}{l}\text { Instructors are aware of and responsive to students' learning } \\
\text { needs }\end{array}$ & 4.2 \\
\hline $\mathbf{3}$ & The instructors are easy to contact & 4.6 \\
\hline $\mathbf{4}$ & The course textbook(s) are helpful in learning concepts & 4.0 \\
\hline $\mathbf{5}$ & $\begin{array}{l}\text { I am comfortable in volunteering my opinion in chats and } \\
\text { threaded discussions }\end{array}$ & 4.6 \\
\hline $\mathbf{6}$ & $\begin{array}{l}\text { I am learning how to think clearly about the course subject } \\
\text { matter }\end{array}$ & 4.6 \\
\hline $\mathbf{7}$ & $\begin{array}{l}\text { I try to think what I am learning in the course from my own } \\
\text { experience }\end{array}$ & 4.5 \\
\hline $\mathbf{8}$ & Overall, the instructors are doing an excellent teaching job & 4.7 \\
\hline $\mathbf{9}$ & The level of detail provided is about right & 4.7 \\
\hline $\mathbf{1 0}$ & Students value each other's opinion & 4.0 \\
\hline $\mathbf{1 1}$ & I am learning a lot from the course & 4.3 \\
\hline $\mathbf{1 2}$ & The course workload is about right & 4.2 \\
\hline $\mathbf{1 3}$ & Case write-ups are available & 4.0 \\
\hline $\mathbf{1 5}$ & Online chat sessions are valuable & 4.1 \\
\hline $\mathbf{1 6}$ & Email is a valuable course tool & 4.3 \\
\hline $\mathbf{1 7}$ & The threaded discussions are useful & 4.2 \\
\hline $\mathbf{1 8}$ & Overall, I have learnt a lot from the course & 4.2 \\
\hline $\mathbf{1 9}$ & iDlystems is user friendly & 3.8 \\
\hline $\mathbf{2 0}$ & My team leader did an excellent job & 3.8 \\
\hline $\mathbf{2 1}$ & Blackboard is user-friendly & 4.3 \\
\hline $\mathbf{2 2}$ & With iDL systems I learnt materials easily & 4.3 \\
\hline $\mathbf{2 3}$ & With Blackboard I learnt materials easily & 4.10 \\
\hline $\mathbf{2 4}$ & I recommend the use of iDL systems only & 2.5 \\
\hline $\mathbf{2 5}$ & I recommend the use of Blackboard only & 4.5 \\
\hline $\mathbf{2 6}$ & I will take more online courses & 4.16 \\
\hline & Overall likeability index & \\
\hline & & \\
\hline
\end{tabular}

Appendix -6

iDLSystems Course Site

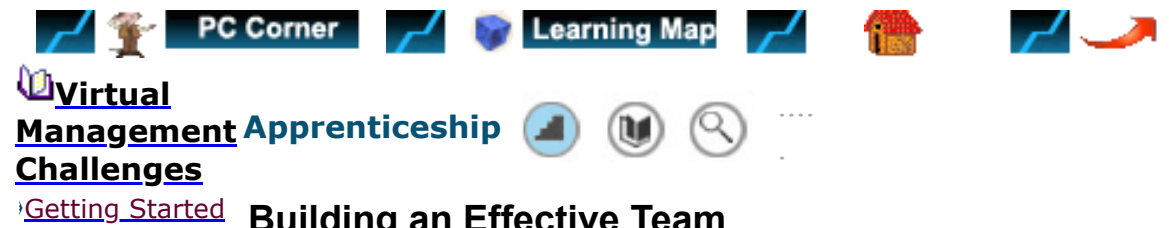

Page 1 of 12 Next

\section{Getting Started Building an Effective Team \\ Introduction}

(Unit 1)

Team

Building. Unit
In this concept we will discuss team, virtual team and building teams.

Virtual groups are becoming more prevalent in the business world. How do these groups differ from those that are face-to-face? How are they similar? 

characteristics of team. Click on the play to listen to the audio. The following pages will explain

Team some of these.

Development

Virtual Team

Activity

\section{Team}

Building.(Unit 2): Quiz

Changes and Challenges in IT

(Unit 3).

Changes and Challenges in IT (Unit 3): Quiz Continuity and Change (Unit 4).

Continuity and Change (Unit 4): Quiz

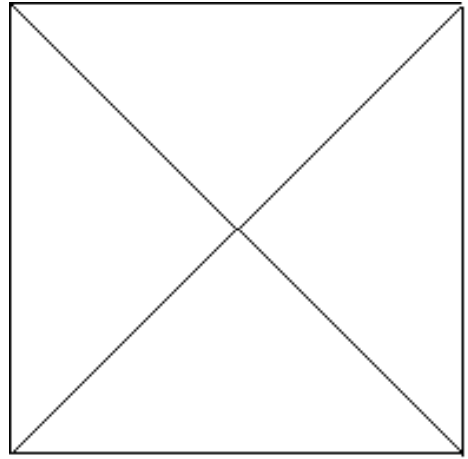

Copyright (c) 2003. iDL Systems. All rights reserved. 